\title{
Diurnal atmospheric extinction over Teide Observatory (Tenerife, Canary Islands)
}

\author{
A. Jiménez ${ }^{1}$, H. Gonzalez Jorge ${ }^{2}$, and M.C. Rabello-Soares ${ }^{1}$ \\ 1 Instituto de Astrofísica de Canarias, E-38200 La Laguna, Tenerife, Spain \\ 2 Departamento de Fisica Fundamental y Experimental, Universidad de la Laguna, Tenerife, Spain
}

Received June 12; accepted October 14, 1997

\begin{abstract}
Data from five continuous years (1984 to 1989) at Teide Observatory (Izaña, Tenerife, Canary Islands) obtained with a full-disc solar multichannel photometer devoted to the detection of solar intensity oscillations has now been used for a detailed study of the extinction coefficient behaviour at several wavelengths (from $450 \mathrm{~nm}$ to $870 \mathrm{~nm}$ ) and aerosol size distributions under different atmospheric conditions. From this study we conclude that an extinction coefficient around $0.075 \mathrm{mag}$ airmass $^{-1}$ at $680 \mathrm{~nm}$ constitutes the borderline between clear days and dusty days affected by the occasional presence of wind-blown Saharan dust. In the former the extinction can vary between 0.04 and $0.07 \mathrm{mag}$ airmass $^{-1}$ (at $680 \mathrm{~nm}$ ) and in the latter between 0.075 and $0.8 \mathrm{mag}$ airmass ${ }^{-1}$ (at $680 \mathrm{~nm}$ ). Moreover the dust produces a grey effect in the wavelength range analysed in this work. From the extinction coefficients we use inversion methods to compute the particle size distribution in both atmospheric conditions. Approximately, the extinction on clear and dusty days is produced by aerosol particles with radius between 40 and $120 \mathrm{~nm}$ and between 120 and $3000 \mathrm{~nm}$, respectively.
\end{abstract}

Key words: atmospheric effects — site testing

\section{Introduction}

Teide Observatory (Tenerife, Canary Islands) and Roque de los Muchachos Observatory (La Palma, Canary Islands), are first-class sites for astronomical observations. Due to the small distance between the two islands (about $150 \mathrm{~km}$ ), their equal altitude and geographical situation, this work is applicable to both islands. The observations used here have been carried out at Teide Observatory, situated at $28^{\circ} 17.8^{\prime} \mathrm{N}$ and $16^{\circ} 29.4^{\prime} \mathrm{W}$ and $350 \mathrm{~km}$ from the northwest coast of Africa. It offers exceptional conditions

Send offprint requests to: A. Jiménez due to the stable subsiding maritime airmass encountered normally above the inversion layer formed between 800 and $1200 \mathrm{~m}$ (McInnes \& Walker 1974). These conditions are typical for isolated mountains on islands. Its altitude, about $2400 \mathrm{~m}$, places the observatory above the inversion layer for a large fraction of the year. During summer occasional presence of wind-borne Saharan dust may occur but is very infrequent during the rest of the year (Sanchez 1970; Murdin 1985). The occasional presence of dust may create difficulties for some observing programmes (where very high transparency is required) but does not spoil the image quality (Brandt \& Wohl 1982) and are excellent for imaging instrumentation. As a result of the excellent conditions and geographic situation, the helioseismology community has also chosen Teide Observatory as a site to take part in worldwide terrestrial network observing programmes, e.g. GONG, IRIS, BISON, TON and LOW-L.

Although several site-testing campaigns have been carried out to characterize the Canarian Observatories putting into evidence their excellent quality (Murdin 1985; Vernin \& Muñoz-Tuñon 1992, 1994; Brand \& Wohl 1992; Hill et al. 1994a,b; Muñoz-Tuñon et al. 1997) none of them have undertaken the study of the behaviour of the extinction coefficients at several wavelengths under different atmospheric conditions. For doing so a large database such as the one used here is necessary. Only using several years of data it is possible to cover the range from excellent "coronal" days where extinction is very low to "absorbent" days where Saharan dust is present in the skies above the island. Since the occasional presence of Saharan dust is unavoidable, information on its effects on astronomical observations are of great utility. A way of obtaining this information is to compare the extinction behaviour and the aerosol size distribution on days with and without dust. These points will be addressed in this paper. 


\section{Instrumentation and observations}

Measurements of solar irradiance are performed simultaneously at four wavelengths. High photometric accuracy is achieved by integration of photodiode signals over 13 seconds, using an ultra-low-noise current-to-voltage amplification stage and a digitization scheme with a voltageto-frequency converter coupled to a high stability time base. The apparatus is equatorially mounted, its tracking is servo-controlled and other parameters such as errors on both axes, temperature measurements at the detectors and at voltage-to-frequency converters are also recorded.

The instrument used for this work is the Solar Luminosity Oscillation Telescope (SLOT), (Andersen et al. 1988a), built at the Space Science Department at ESTEC and devoted to the detection of solar intensity oscillations (Jiménez et al. 1987, 1988, 1990) over five continuous years (1984 to 1989) and for a seasonal atmospheric conditions study (Andersen et al. 1988b). During the campaign, the sets of filters were changed four times to check different observing possibilities and improve the detection of solar intensity oscillations. Table 1 shows filter sets used during the campaign. Nine different filters were used, but only 5 have been used in the present analysis: $680 \mathrm{~nm}$ (common to all filter sets), $500 \mathrm{~nm}, 450 \mathrm{~nm}$, $870 \mathrm{~nm}$ and $770.2 \mathrm{~nm}$. Channel \#4 of filter set \#1, at $680 \mathrm{~nm}$, was a redundant channel to assess the instrumental noise. Channel \#2 with filter sets \#1 and \#2 at $1060 \mathrm{~nm}$ presented a low frequency trend due to temperature variation because the photometer lacked a cooling system needed for near infrared measurements. Channel \#2 with filter sets \#3 and \#4 at $517.8 \mathrm{~nm}$ and channel \#3 of filter set \#3 at $516.2 \mathrm{~nm}$ were very similar, the only difference was in their bandwidths. This was an attempt to increase the signal-to-noise ratio by dividing the signal in both channels (as suggested by Harvey 1986). For atmospheric extinction analysis, the behaviour of these channels is the same as the one at $500 \mathrm{~nm}$ (channel \#3 with filter set \#1 and \#2) which has been used in this work.

\section{Data reduction and analysis}

Figure 1 shows two days observed with filter set\# 4, where a) corresponds to a clear day and b) to a dusty day. The typical shape of the curves are due to the well known effect of atmospheric extinction. The observed intensity $I$ is given by:

$I=I_{0} \mathrm{e}^{-K_{\lambda} M(Z)}$,

where $I_{0}$ is the intensity above the terrestrial atmosphere, $M(Z)$ the airmass to be traversed by sunlight before reaching the photometer (normalized to unity at zenith), and $K_{\lambda}$ the atmospheric extinction coefficient at $\lambda$. Representing the photometric measurements by its instrumental magnitude defined in the standard astronomi- cal way, $m=-2.5 \log I$, we find the well known Bouguer law:

$m=m_{0}+1.086 K_{\lambda} M(Z)$.

A linear fit to $m-M(Z)$ gives the extinction coefficient $K_{\lambda}$ during the time span used.

Before any fitting procedure was applied, data were selected to avoid days where strong transparency changes occurred, i.e. when dust appeared during the day making it partially clear and partially dusty, or vice versa when dust moved away from the observatory during the day. These days yield a variable extinction coefficient which is the result of mixed atmospheric conditions. In addition, days with early shutdown or late startup, power failure or other technical problems, uncompleted days or days with a large fraction of data gaps, have also been discarded. Because to this stringent selection, only 650 days approximately of the 1730 days of the whole campaign were selected for this work. For this reason, any percentage of clear or dusty days obtained with the data used here have no statistical significance.

In the analysis, each day is divided taking 4.5 hours before and after local noon. Points at the beginning and at the end of the day are rejected because the airmass computation is less accurate. After logarithmic transformation, the magnitude as a function of airmass (Bemporad formula, Golay 1974) for the two halves of the day are fitted to straight lines using a least-squares fitting procedure. This yields two extinction coefficients per day per channel together with the standard deviation of the fit which provides information about the dispersion of the points. In this way we separate the effect of the extinction coefficients during the afternoon that usually are $7 \%$ lower (Andersen et al. 1988b) than in the morning. This difference is believe to be caused by the influence of the African continent or by local convection effects. The extinction coefficients may also vary as a function of time during the morning and afternoon hours, this effect will be discuss at the end of Sect. 4.

\section{Extinction coefficient behaviour with wavelength}

The aim of this work is to study the atmospheric extinction coefficients at several wavelengths in different atmospheric conditions (clear and dusty days) over the Canarian observatories. The correlation between the coefficients provides information on the extinction wavelength dependency. If we represent the $K_{\lambda_{1}}$ versus $K_{\lambda_{2}}$ slopes equal to unity imply a grey behaviour. Figure 2 shows the extinction coefficients at several wavelengths versus the one at $680 \mathrm{~nm}$. Looking at this figure some features are immediately apparent. The functions $K_{\lambda_{1}}=f\left(K_{680}\right)$ are straight lines but at low values of $K_{680}$ the slope changes. This is clearly seen mainly at $450 \mathrm{~nm}$ and $500 \mathrm{~nm}$. To verify this and calculate the value at which the slope 
Table 1. Filters sets used during the whole campaign. $\lambda_{\mathrm{c}}$ and $\Delta$ are the central wavelength and band width of each filter

\begin{tabular}{|c|cccc|}
\hline & $\begin{array}{c}\text { Filter set 1 } \\
\text { (84 Aug. / 85 Jun.) }\end{array}$ & $\begin{array}{c}\text { Filter set 2 } \\
\text { (85 Jun. / 87 Aug.) }\end{array}$ & $\begin{array}{c}\text { Filter set 3 } \\
\text { (87 Aug. / 88 Dec.) }\end{array}$ & $\begin{array}{c}\text { Filter set 4 } \\
\text { (88 Dec. / 89 Jun.) }\end{array}$ \\
\hline \hline Channel & $\lambda_{\mathrm{c}}(\Delta) \mathrm{nm}$ & $\lambda_{\mathrm{c}}(\Delta) \mathrm{nm}$ & $\lambda_{\mathrm{c}}(\Delta) \mathrm{nm}$ & $\lambda_{\mathrm{c}}(\Delta) \mathrm{nm}$ \\
\hline $\mathbf{1}$ & $680(10)$ & $680(10)$ & $680(10)$ & $680(10)$ \\
$\mathbf{2}$ & $1060(10)$ & $1060(10)$ & $517.8(3.5)$ & $517.8(3.5)$ \\
$\mathbf{3}$ & $500(10)$ & $500(10)$ & $516.2(9.1)$ & $450(10)$ \\
$\mathbf{4}$ & $680(10)$ & $870(10)$ & $770.2(4.7)$ & $770.2(4.7)$ \\
\hline
\end{tabular}

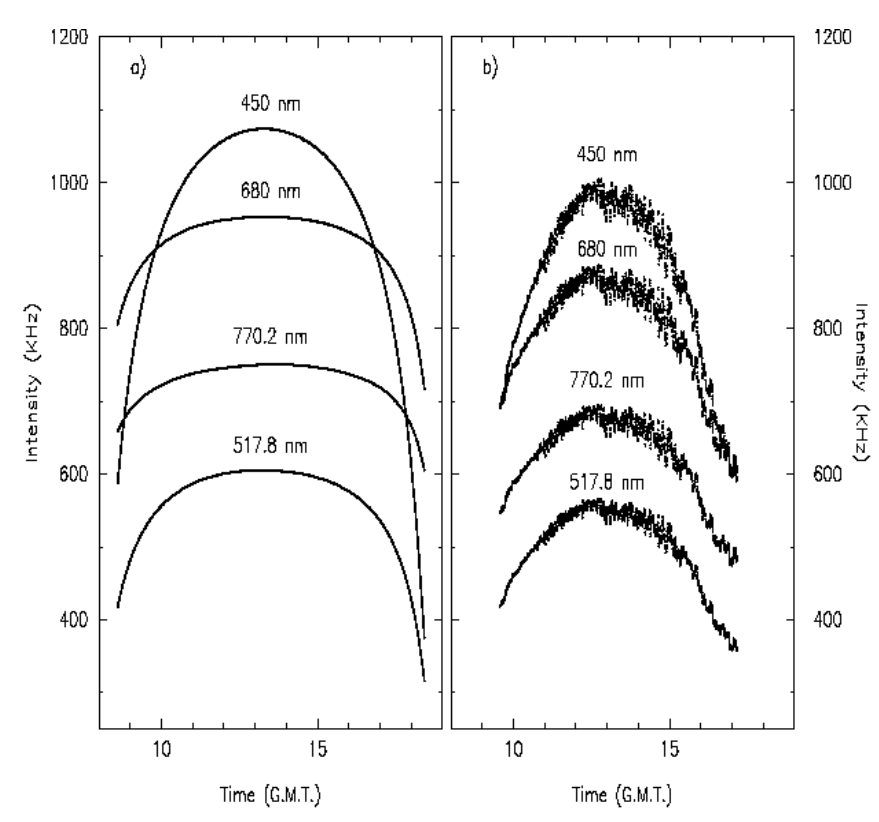

Fig. 1. Examples of two days observed corresponding to filter set \# 4 (see Table 1), a) corresponds to a clear day and b) to a dusty day

changes we do the following. For each one of the extinction coefficients pairs in Fig. 2, we fit 9 straight lines covering different but overlapping regions on the $x$-axis. For $450 \mathrm{~nm}$ versus $680 \mathrm{~nm}$, only 5 straight lines were fitted due to the lower number of points, mainly at high extinction values. Table 2 shows the intervals and the number of points used in the fits. The fitting procedure is a standard least-squares method with errors in both coordinates, which we obtain from the computation of extinction coefficient.

Figure 3 shows the $K_{\lambda}$ values with the straight lines fitted to the different overlapping regions. The slopes obtained in these regions are plotted in the insets. The ordinates of the insets correspond to the slopes and the abscissae represent the regions at which these slopes are obtained. A point with abscissa of 0.3 means that the slope

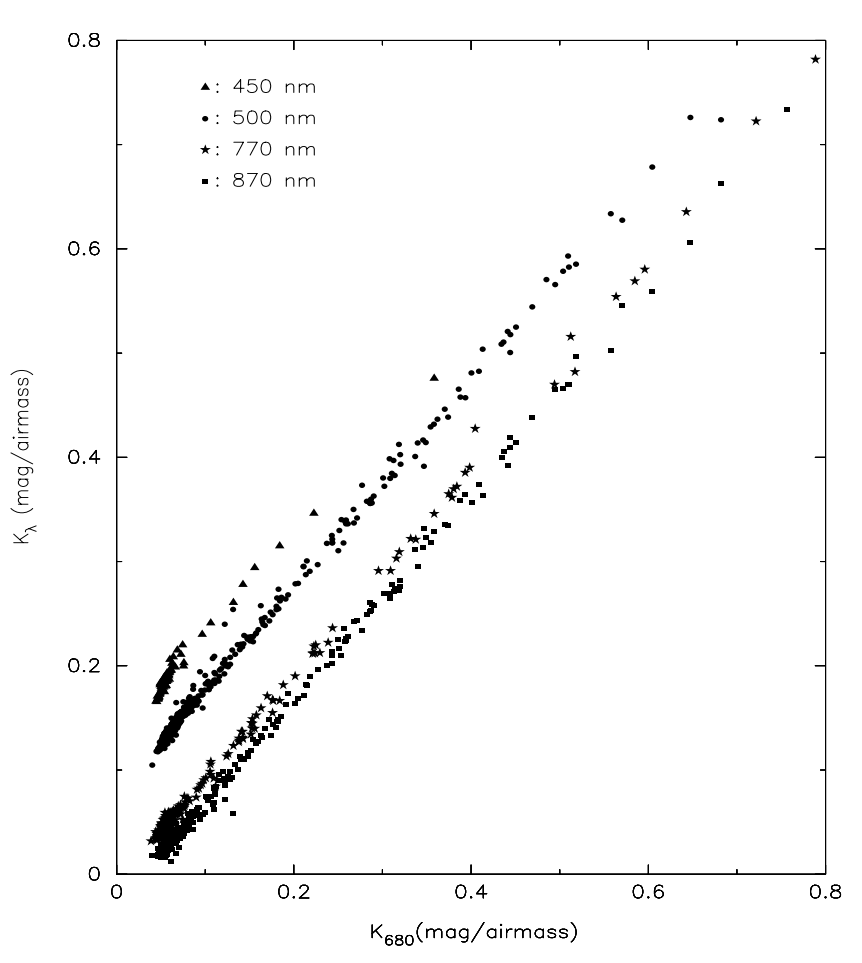

Fig. 2. Extinction coefficients (mag airmass ${ }^{-1}$ ) for all the wavelengths used in this work versus the one at the common channel to all the observations $(680 \mathrm{~nm})$. Notice the slope changes for extinction coefficients smaller that $0.1 \mathrm{mag}$ airmass $^{-1}$

has been obtained from points with abscissae between 0.3 and 0.8 mag airmass $^{-1}$; a point with abscissa 0.2 has been obtained from 0.2 to 0.8 mag airmass ${ }^{-1}$, and so on (see Table 2). Therefore there are 9 points (determination of the slopes) in the insets, except for $K_{450}$ (Fig. 3a) in which there are only 5 .

The slopes of the straight lines are close to 1 for the high extinction values, but points with low extinction values have higher slopes. In addition the increase of the 
Table 2. Number of points $(N)$ used in each of the linear fits to the lines $K=a+b K_{680}$ on several and overlapping regions values of $K_{680}$ (see text)

\begin{tabular}{|l|cccc|}
\hline $\begin{array}{l}\text { Region } K_{680} \\
\left(\text { mag airmass }^{-1}\right)\end{array}$ & $\begin{array}{c}\text { No. of points for } \\
\lambda=450 \mathrm{~nm}\end{array}$ & $\begin{array}{c}\text { No. of points for } \\
\lambda=500 \mathrm{~nm}\end{array}$ & $\begin{array}{c}\text { No. of points for } \\
\lambda=770 \mathrm{~nm}\end{array}$ & $\begin{array}{c}\text { No. of points for } \\
\lambda=870 \mathrm{~nm}\end{array}$ \\
\hline \hline $\mathbf{0 . 3}-\mathbf{0 . 8}$ & - & 45 & 22 & 41 \\
$\mathbf{0 . 2}-\mathbf{0 . 8}$ & - & 76 & 32 & 70 \\
$\mathbf{0 . 1}-\mathbf{0 . 8}$ & - & 144 & 63 & 123 \\
$\mathbf{0 . 0 9}-\mathbf{0 . 8}$ & - & 158 & 68 & 131 \\
$\mathbf{0 . 0 8}-\mathbf{0 . 8}$ & 8 & 175 & 71 & 144 \\
$\mathbf{0 . 0 7}-\mathbf{0 . 8}$ & 12 & 213 & 84 & 165 \\
$\mathbf{0 . 0 6}-\mathbf{0 . 8}$ & 20 & 392 & 121 & 250 \\
$\mathbf{0 . 0 5}-\mathbf{0 . 8}$ & 69 & 667 & 277 & 488 \\
$\mathbf{0 . 0 0}-\mathbf{0 . 8}$ & 87 & 713 & 343 & 533 \\
\hline
\end{tabular}

slopes is smaller as we go to higher wavelengths and slopes are always close to one for $K_{870}$ versus $K_{680}$.

The dependence on wavelength of the atmospheric aerosol extinction coefficient for visible light is in good agreement with the Ångstrom formula (see for example Cachorro et al. 1989):

$K_{\lambda}=\beta \lambda^{-\alpha}$,

where $\beta$ is the turbidity coefficient and $\alpha$ is the Angstrom exponent. This formula is usually written as:

$\log K_{\lambda}=\log \beta-\alpha \log \lambda$

with $\alpha$ being the slope of a straight line. From the logarithm scale, it can be seen that the extinction coefficient varies less between the two longest wavelengths of the interval than between the two smallest. This explains why the slopes for 870 and $770 \mathrm{~nm}$ are much smaller than the slopes at 450 and $550 \mathrm{~nm}$ in Fig. 3. On the other hand, the fact that the slopes are equal to 1 for only the higher extinction values indicates from (3) that $\alpha \simeq 0$, which is a value of $\alpha$ related to aerosol particles of the dust size. When other points of lower $K_{\lambda}$ are introduced into the fits, $\alpha$ needs to increase to lead higher values of the slopes and, $\alpha$ increases as aerosol sizes decrease. Therefore, this different behaviour of the slopes is produced by the effect of Saharan dust.

The first evidence of this effect is based on the long experience of our observers. In all our observing campaigns, observers fill in a detailed daily running logbook in which all the observing conditions are recorded. In particular, the quality of the day is classified as coronal, pure, diffuse and absorbent (dusty). When there is a considerable amount of dust in the sky, it is easy to evaluate its quality as the inhabitants of the islands know very well. When the sky is blue and clear is also easy to classify it as a coronal or pure day (in this work termed "clear" days). The classification problem arises when the day is somewhat diffuse and it is not possible to know if it is produced by water vapour, aerosols, etc. A detailed comparison of notes with their respective extinction coefficients shows that on clear days (annotated as coronal-pure) the extinction values at $680 \mathrm{~nm}$ are never higher than about $0.07-0.09 \mathrm{mag}$ airmass $^{-1}$, while on dusty days (diffuse-absorbent) they are always higher. The classification of the days based on our observers' skills, is absolutely valid for clear and dusty skies, but ambiguous for the extinction coefficient range between about $0.07-0.1 \mathrm{mag} /$ airmass at $680 \mathrm{~nm}$. To estimate the extinction coefficient at which the effect of the dust starts to appear, we have performed a more objective calculation.

On dusty days, the standard deviation (sigma) of the daily linear fit increases (see Fig. 1b). Using the sigma of the fits, it is possible to see the effects of the dust in more detail. In Fig. 4 we show $K_{680}$ divided by the standard deviation of the Bouguer law fit (sigma), versus $K_{680}$. As is clearly seen in this figure, at low $K_{680}$, the $K_{680}$ /sigma ratio is concentrated in a narrow vertical band, whereas for high extinction values it is not (low broad horizontal). The first band corresponds to clear days with low extinction and low sigma; of course, the quality of clear days is not always the same and this produces the large spread of points on the $y$-axis. The second band corresponds to dusty days with high extinction values but also with high dispersion. Our problem is to find the $K_{680}$ value threshold that can separate both. The inset of Fig. 4 shows the region corresponding to this "transition" band where it seems that the separation between the two bands could be around 0.075 mag airmass $^{-1}$ (at $680 \mathrm{~nm}$ ) in agreement with the experience of our observers. The exact value $K_{680}$ at which the slope change occurs can be somewhat affected by the selection of the days in the analysis. If all the days had been used, very probably this point would have been ill defined as the amount of "intermediate" $K$ values would have increased. However this would only affect the precision at which this value is known.

Therefore, we choose this threshold value as a border point at which the effects of the dust begin to be significant. Probably with this classification, a small amount 


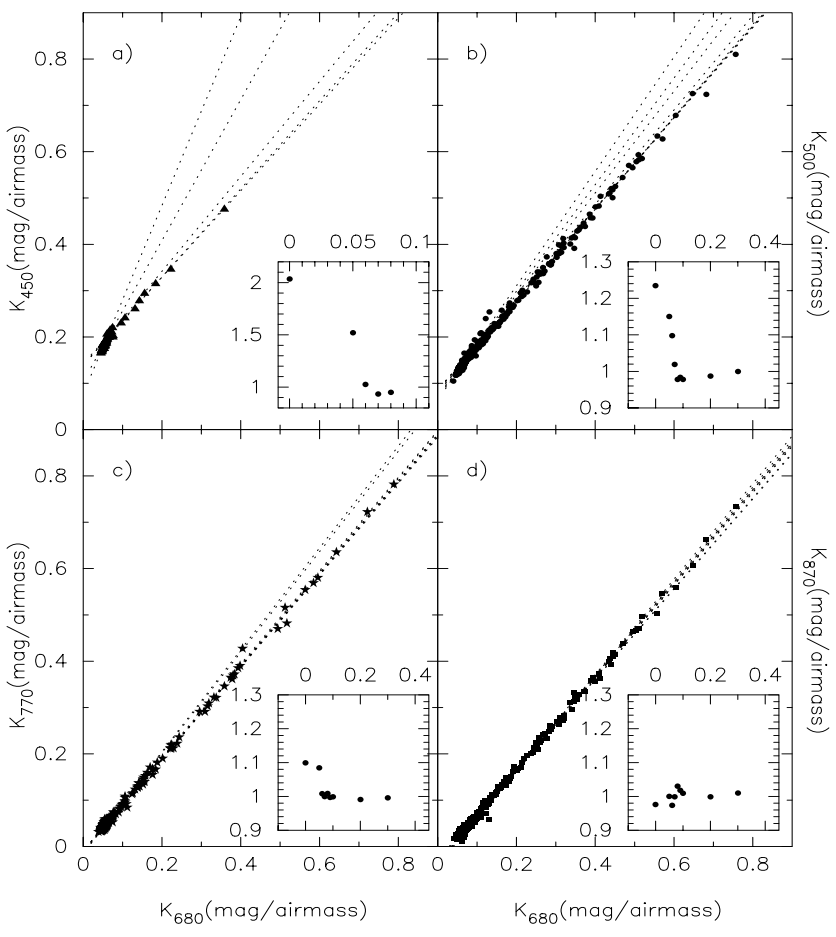

Fig. 3. Extinction coefficients (mag airmass ${ }^{-1}$ ) of Fig. 2 plotted separately and fitted to straight lines for several overlapping regions values of $K_{680}$ as explained in the text (see Table 2). In the insets are shown the slopes of the fitted lines (see text)

of points could be swapped between the two bands. This value of $0.075 \mathrm{mag}$ airmass $^{-1}$ (at $680 \mathrm{~nm}$ ) really represents an inferior limit for the selection of clear days and it can be increased at least up to $0.09 \mathrm{mag}$ airmass $^{-1}$ (at $680 \mathrm{~nm}$; a test made changing the value of the borderline between $0.07-0.09 \mathrm{mag}$ airmass ${ }^{-1}$ shows that the result does not change significantly). On this basis, we divide each of the four extinction curves of Fig. 3 in two: extinction values lower and higher that 0.075 mag airmass ${ }^{-1}$ (at $680 \mathrm{~nm}$ ). Then straight lines are fitted to each of the 8 curves as explained before. Figure 5 shows this analysis and Table 3 the slopes obtained in the fits. From Fig. 5 and Table 3 it seems to be clear that the Saharan dust at Teide Observatory has a grey behaviour in the wavelength used in this work (this result was first proposed by Whittet et al. 1987 for Roque de los Muchachos Observatory, La Palma, Canary Islands).

As we mentioned at the end of Sect. 3, we will discuss now the effect of neglecting the temporal variation of the extinction as function of time. Computing the extinction coefficients in the morning and in the afternoon

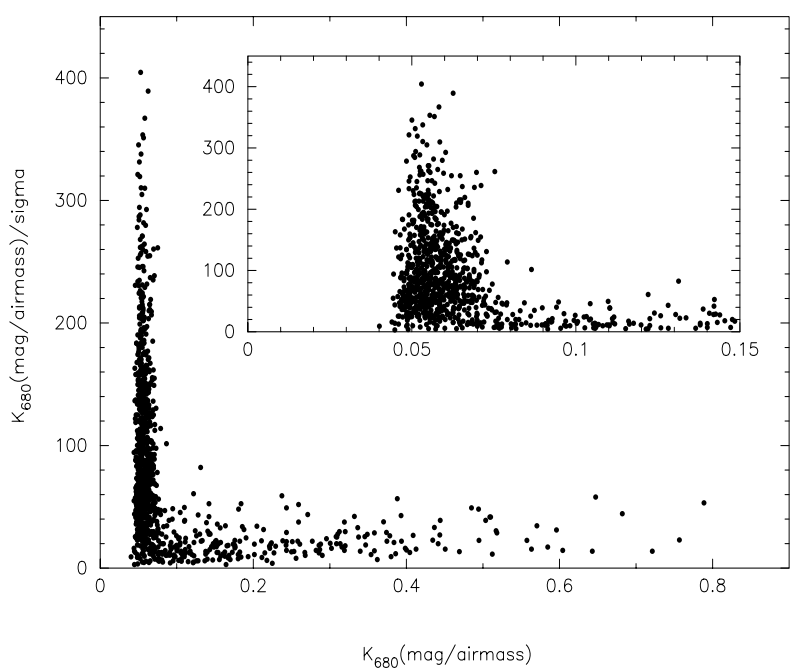

Fig. 4. Extinction coefficient at $680 \mathrm{~nm}$ divided by its sigma value obtained from the Bouguer law fit versus extinction at $680 \mathrm{~nm}$. The two bands (narrow vertical and spread horizontal) corresponds to clear and dusty days, respectively. In the inset, the interval corresponding to the "transition" region between the two bands

instead of, for example, every hour, we are obtaining an average of the extinction during that half of the day. If we compute the extinction every hour we would get 4 or 5 values around the value obtained using the half day (this have been checked for several days). As the days have been separated into clear and dusty days with no atmospheric mixing conditions, the temporal values of the extinction would be on the straight lines of Fig. 5 (left panel) for clear days and in Fig. 5 (right panel) for dusty days. In conclusion, if we had obtained the extinction values on a shorter time basis, one hour for example, we would have 4 or 5 times more points in the figures but our results would be the same.

Table 3. Slopes of the lines $K_{\lambda}=a+b K_{680}$ corresponding to Figs. 5a and 5b) (see text)

\begin{tabular}{|l|cc|}
\hline$\lambda(\mathrm{nm})$ & Clear days $(\mathrm{a})$ & Dusty days $(\mathrm{b})$ \\
\hline \hline $\mathbf{4 5 0}$ & $2.132(+/-) 0.105$ & $1.028(+/-) 0.042$ \\
$\mathbf{5 0 0}$ & $1.333(+/-) 0.025$ & $1.011(+/-) 0.015$ \\
$\mathbf{7 7 0}$ & $1.159(+/-) 0.033$ & $0.999(+/-) 0.009$ \\
$\mathbf{8 7 0}$ & $1.026(+/-) 0.029$ & $1.009(+/-) 0.012$ \\
\hline
\end{tabular}




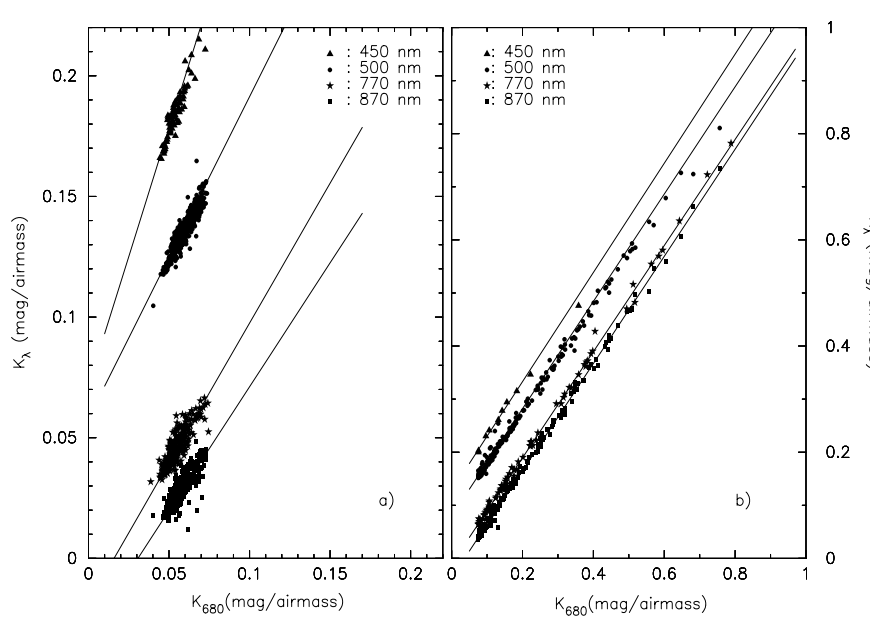

Fig. 5. Extinction coefficients together with the best linear fits, once the days have been separated in clear days (extinction at $680 \mathrm{~nm}<0.075 \mathrm{mag}^{2}$ airmass ${ }^{-1}$ ) and dusty days (extinction at $680 \mathrm{~nm}>0.075 \mathrm{mag}$ airmass $^{-1}$ ). See Table 3

\section{Theoretical approach to the extinction coefficient dependency on wavelength at Teide Observatory}

The fact that the slopes in Fig. 5 are different for Saharan dust and for non-Saharan dust episodes means that the aerosol particles have at least different size distributions. Using Mie theory, the extinction coefficient may be defined as

$K_{\lambda}(\lambda, m)=\int_{0}^{\infty} \pi r^{2} Q_{\mathrm{e}}(r, \lambda, m) n(r) \mathrm{d} r$,

where $Q_{\mathrm{e}}(r, \lambda, m)$ is the extinction efficiency factor, $n(r)$ the particle size distribution in the atmospheric column, $r$ the particle radius, $m$ the particle refractive index, and $\lambda$ the wavelength of the incident light.We computed the extinction efficiency factors for the wavelengths used in this work using the Mie scattering code of Wiscombe (1980) and obtained Fig. 6 . The difference between the extinction efficiency factors at two different wavelengths is just a shift in particle sizes as shown in Fig. 6a. There is a particle radius for which the efficiency factors have reached the value of 2 at all wavelengths. Particles with this and larger radii contribute to the extinction coefficient in the same quantity regardless of the wavelength. The radius interval where the extinction coefficients vary with wavelength can be determined by applying the criterion of Heintzenberg et al. (1981), who represented the ratios of the extinction efficiency factors at different wavelengths as a function of particle radius. Then, the extinction coefficients are wavelength dependent (slopes different from unity) if the aerosol particle sizes are in the radial interval where these ratios are not constant. Also, the extinction coefficient is not wavelength dependent (slopes equal to unity) if the aerosol particle sizes are outside that interval and consequently the ratios are constant. Note that the dependence on wavelength is an effect of the independent information content in a set of multi-wavelength optical data. Figure $6 \mathrm{~b}$ shows the criterion of Heintzenberg et al. (1981) applied to the wavelengths 450, 770 and $870 \mathrm{~nm}$, for the complex refractive index of $1.55-0.005 i$ $(i=\sqrt{-1})$. Fouquart et al. (1987), Carlson \& Benjamin (1980), and Patterson (1977) considered this refractive index for Saharan dust aerosols. From Fig. 6b, the particles with sizes around $100 \mathrm{~nm}$, lead to extinction coefficients that show wavelength dependence.

\subsection{Extinction coefficients computed for known aerosol size distributions}

This section shows that the extinction coefficient wavelength dependence is in good agreement with the criterion of Heintzenberg et al. (1981). Both Saharan and non- Saharan aerosol conditions are simulated to show how dust particles cause the non-wavelength dependency. Atmospheric aerosol size distributions are characterized by a multi-modal model, and each mode is represented by a lognormal distribution. We will consider the following three modes: (1) the "accumulation mode" with the smallest particle sizes, (2) the "coarse mode" with larger sizes than the accumulation mode, and (3) the "dust mode" with the largest sizes. Aerosol size distributions have not been measured at Teide Observatory on a long-term basis, but Saharan dust optical properties and size distributions have been studied in many African locations in recent years. D'almeida (1987) measured the size distribution in Timbuctu and in Agadez for three different weather regimes: 1 . "desert background", when aerosols are present at a relative low concentration for the site; 2 . "desert with wind carrying dust", when some dust aerosol particles are transported from the desert and added to the background aerosols, and 3. "sand storm", high concentrations of dust particles come directly from the desert. Trimodal lognormal size distributions were then fitted to the measured data. Obviously, the size distribution measured during a sand storm is not representative of Teide Observatory aerosol size distributions because most of the larger particles are not transported very far. Therefore, we assumed only the "desert background" and the "desert with wind carrying dust" size distributions to calculate approximate values of the extinction coefficients for Saharan dust episodes at Teide Observatory. The third mode was used in the study for further clarification. The refractive index assumed in this case was $1.55-0.005 i$.

To represent a non-Saharan episode, we assumed size distributions from Whitby (1978), which are derived from averages of measurements on sites of the same category. We chose the categories representative of "clear day 

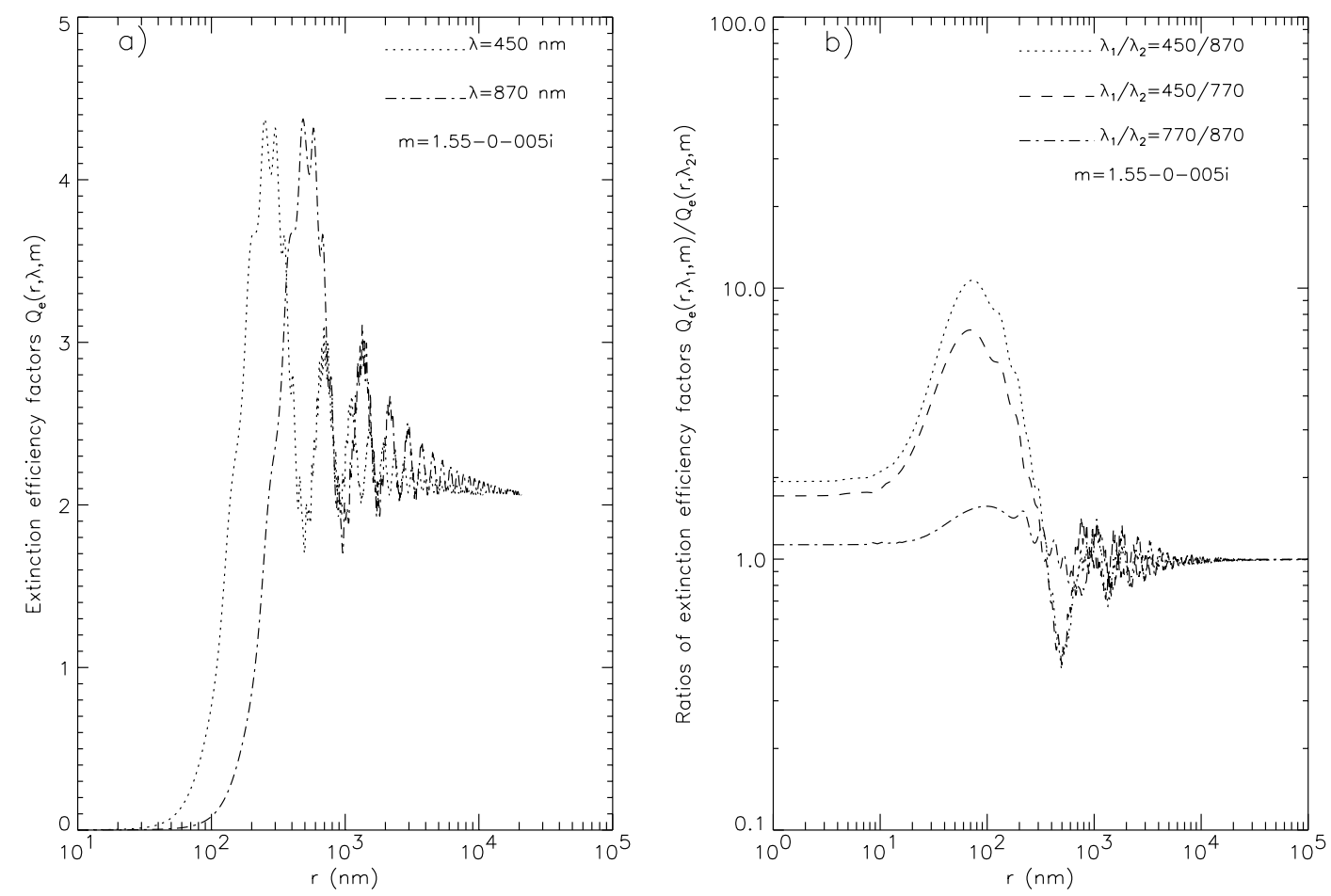

Fig. 6. a) Extinction efficiency factors at 450 and $870 \mathrm{~nm}$ for a refractive index of $1.55-0.005 i$. Note that both curves are exactly the same except for one being shifted from the other. b) Ratios of extinction efficiency factors for selecting the interval of radius where the extinction coefficients can show wavelength dependence

background" and "polluted clear days". We assumed a refractive index of 1.45 , which is representative of sulphuric acid components (Palmer \& Williams 1975) at 20\% relative humidity (Charlson 1978). This relative humidity is in the interval of relative humidities measured at Teide Observatory. A layer $1 \mathrm{~km}$ thick was considered in both cases. The other atmospheric layers were considered to contribute to the extinction coefficients similarly in both conditions, with and without Saharan dust. We seek particle sizes that affect the extinction coefficient dependency with wavelength and consider only spherical particles using Mie theory. Figure 7a shows these four size distributions and Fig. 7b the corresponding extinction coefficients computed using the distributions of Fig. 7a. In Fig. 7b we have also included two more results obtained for Saharan dust episodes but using only the "accumulation mode": "accumulation mode of desert with wind carrying dust" and "accumulation mode of desert background". Also to estimate the influence of the "dust mode", we calculated the extinction coefficient for Saharan dust episodes but only for the accumulation and coarse modes (not plotted in Fig. 7b) concluding that the third mode contribution ("dust mode") to the extinction coefficients was only of about $1 \%$. Also extinction coefficients calculated considering both the whole size distribution and only the accumulation and coarse mode, were not wavelength dependent for Saharan dust episodes, and the extinction coefficients calculated for just the accumulation mode of the size distribution showed wavelength dependence. On the other hand, the extinction coefficients calculated for the Whitby size distributions ("clear day background") showed wavelength dependence either way, considering the whole size distribution and only the accumulation mode. It can also be concluded from Fig. 7 that: (1) The aerosol particles in the first mode of all the size distributions considered (Fig. 7a), yield extinction coefficients that are dependent on wavelength. The radius range containing the independent information in the wavelengths is around $100 \mathrm{~nm}$. But since the efficiency factors of particles with radii smaller than $20 \mathrm{~nm}$ is close to zero (see Fig. 6a), their contribution to the extinction coefficient is negligible. Therefore, these smallest sizes of the accumulation mode do not make any difference to the slopes of Fig. 5. (2) The large number of particles with radii over $2000 \mathrm{~nm}$ approximately, with large volume, makes the extinction coefficients independent of wavelength in the Saharan dust cases. (3) In the non-Saharan dust cases, the small number of particles with radius larger than $2000 \mathrm{~nm}$ is not enough to make the extinction coefficients independent of wavelength. Hence, only the particles with a radius of about $100 \mathrm{~nm}$ lead to the wavelength dependence of the extinction coefficients, 

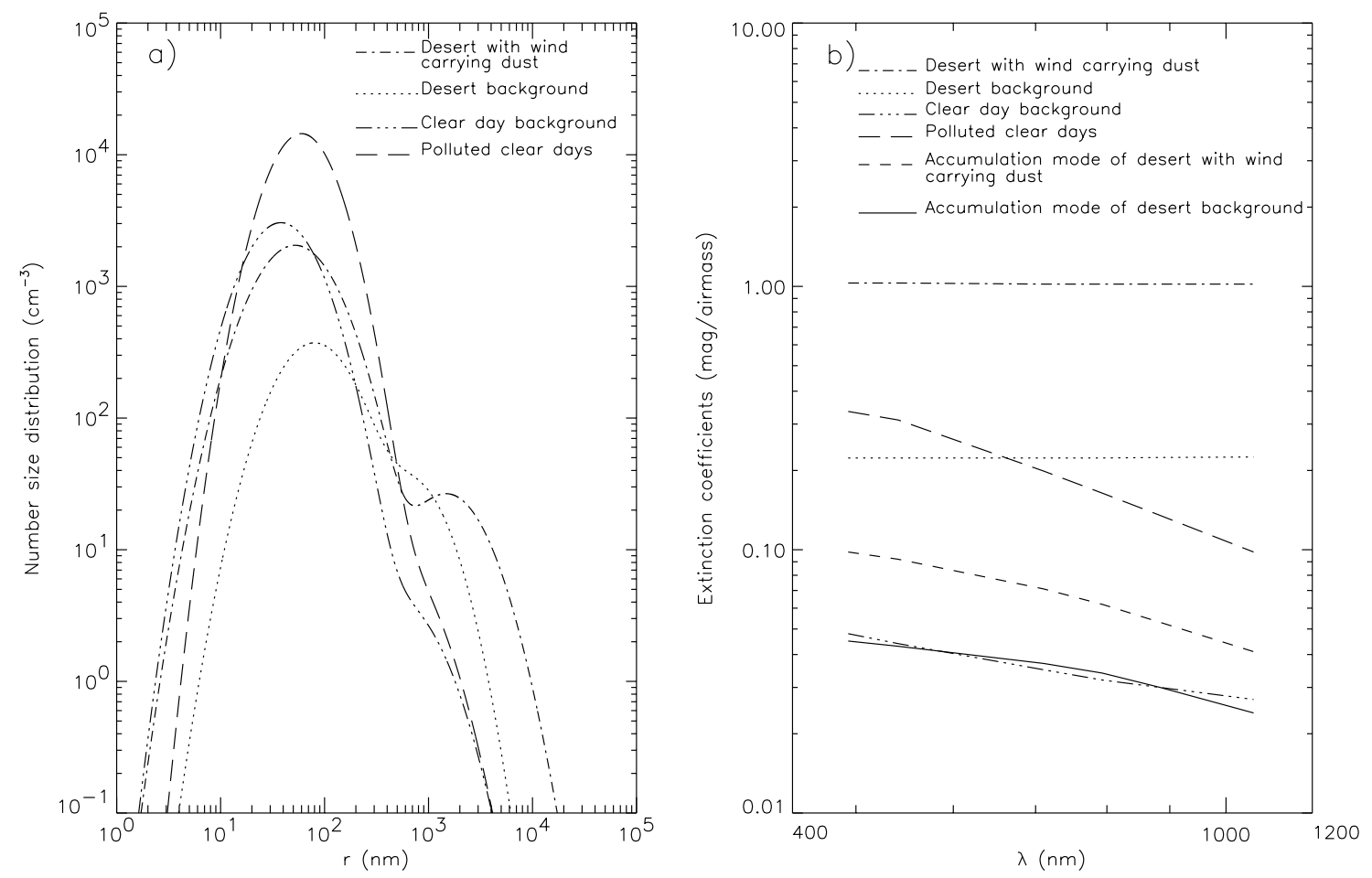

Fig. 7. a) Known aerosol size distribution in different conditions taken from the literature and used in this article. b) Extinction coefficients computed using Mie theory for the whole size distribution of a) and in two cases only for the accumulation mode (see text)

as was concluded from the criterion of Heintzenberg et al. (1981).

\subsection{Computation of aerosol size distributions from the extinction coefficients at Teide Observatory: The inversion problem}

\subsubsection{Uncertainties in the inversion procedures}

Before obtaining the aerosol size distribution from the measured extinction coefficient at Teide Observatory, we introduce the limitations of the method.

Aerosol size distributions can be obtained from inversion of multi-wavelength extinction coefficients (i.e. inversion of Eq. (1)) using the inversion algorithm of King et al. (1978). However, the inversion problem is ill-posed and there are many solutions that reproduce the input data with an error smaller than $0.1 \%$. The assumptions needed in the inversion are the assumptions of Mie theory that considers spherical particles, the aerosol composition through the refractive index, and the radius range for which, the spectral optical depths contain independent information of the particle sizes. These assumptions lead to many uncertainties in the inversion solutions (Gonzalez Jorge \& Ogren 1996). (1) Dust aerosols are rarely spherical particles, Heintzenberg (1978) concluded that applying Mie theory to non-spherical particles systematically distorts the size distribution leading to a shift in the concentration maximum to smaller sizes. (2) The assumptions of a wrong refractive index slightly alter the shape of the retrieved size distribution. In this case, the maximum of the retrieved size distribution modes decreases as the assumed real part of the refractive index increases with respect to the real one. Depending on the assumed refractive index, 1.45 or $1.55-0.005 i$, the retrieved modes have more or fewer particles, respectively. (3) To obtain the size distribution with the inversion method, a finite size range is required. We chose the range between 20 and $3000 \mathrm{~nm}$ because it covers the largest possible size range in which particles can lead to extinction coefficients with wavelength dependence. This finite size range ignores the contribution of the larger particles to the extinction coefficients. As a consequence, extra particles may be placed around $3000 \mathrm{~nm}$. Also, extra particles may appear around the minimum between two retrieved modes or around $20 \mathrm{~nm}$. To increase the selected size range the wavelength interval needs to be extended (1) to the UV to retrieve smaller sizes, and (2) to the IR to retrieve larger particles. The finite size range may lead to errors of about $80 \%$, $30 \%$ and $30 \%$ in the retrieved total number of particles $\left(\int_{0}^{\infty} n(r) \mathrm{d} r\right)$, total surface area $\left(\int_{0}^{\infty} 4 \pi r^{2} n(r) \mathrm{d} r\right)$, and total volume $\left(\int_{0}^{\infty}(4 / 3) \pi r^{3} n(r) \mathrm{d} r\right)$, respectively (Gonzalez Jorge \& Ogren 1996). The large error in the total number 
of particles is due to the size distribution truncation for sizes smaller than $20 \mathrm{~nm}$. Since these particles contribute little to the total surface area and the total volume, not including them in the retrievals yields smaller errors in the retrieved total surface area and total volume than in the retrieved total number concentration of particles. For this reason we will represent the retrieved aerosol size distributions measured at Teide Observatory as volume size distribution (i.e., total volume of particles at each radius range $\left.V(r)=(4 / 3) \pi r^{3} n(r)\right)$.

\subsubsection{Size distributions retrieved from the extinction coefficients measured at Teide Observatory}

For the purpose of this section we need to select the extinction coefficient values to be inverted. One of the more important parameters for the inversion is the number of different wavelengths simultaneously available. As explained in Sect. 2, the aim of the observational campaign was not this kind of study but helioseismology. Because to this, the set of filters was changed several times during the whole campaign. The five wavelengths we are using in this work were never simultaneous, only two by two or three by three and at different periods. Since the inversion algorithm gives better results using more than three wavelengths, we have created the extinction coefficients based on the straight-line fit of Fig. 5 (Table 3 ) to the real data. To compute the extinction coefficients for "clear days", we use the straight lines of Fig. $5 \mathrm{a}$, we select extinction values for $680 \mathrm{~nm}$ from 0.01 to 0.06 in steps of 0.01 and compute the corresponding to the other four wavelengths using the straight lines. For "dust" episodes we do the same but using the straight lines of Fig. $5 \mathrm{~b}$ and choosing the extinction values for $680 \mathrm{~nm}$ from 0.1 to 0.8 with steps of 0.1 .

For the inversions we assumed the size range from $20 \mathrm{~nm}$ to $3000 \mathrm{~nm}$, and the refractive indices $1.55-0.005 i$ and 1.45 for Saharan and non-Saharan episodes, respectively. Figure 8 shows the result for "clear days" and Fig. 9 for "dust" episodes. Note in Fig. 8 how the maximum of the accumulation mode volume for particles with radii below $80 \mathrm{~nm}$ decreases as the extinction coefficient increases, whereas it stays approximately constant for particles with radii between 80 and $100 \mathrm{~nm}$. For particles larger than $100 \mathrm{~nm}$ the volume increases, these particles being responsible for the increase of the extinction coefficient even when their volume is not too high because the extinction efficiency factor increases very rapidly over this range of radii (Fig. 6a). In Fig. 9 the maximum of the coarse-mode volume increases with increasing extinction coefficients for Saharan dust episodes. Clearly, size distributions retrieved from extinction coefficients during Saharan dust have larger volume concentration at the coarse sizes than size distributions during other situations, as expected from the extinction coefficient wavelength dependence. The fact that all the changes in the coarse mode with increasing ex- tinction coefficient are increases in the volume maximum and not shifts of the mode to larger sizes can be a result of using a size range limited from 20 to $3000 \mathrm{~nm}$ and the extinction coefficients computed from the straight-line fits. This last is an effect of constraining all the extinction coefficients to have exactly the same wavelength dependence, which means constraining the retrievals to vary the number of particles but not their sizes. It should not be ignored that the extinction coefficients are not exactly on the fitted lines, and a change in the extinction coefficients could be explained also as a shift of the coarse-mode maximum to larger sizes.

From the retrieved size distributions for $K_{680}=0.06$ (Fig. 8) and $K_{680}=0.1$ (Fig. 9) it might be inferred that the transition between no dust and dust episodes is straightforward. However, the retrieved size distributions assuming the refractive index of $1.55-0.005 i$ for $K_{680}=0.09$ (Saharan dust conditions) had less coarse particles than the retrieved size distributions assuming 1.45 for $K_{680}=0.07$ (non-Saharan dust conditions). This discrepancy was related to the refractive index assumption. The change in the aerosol composition during the dust invasion is gradual as is the change in the extinction coefficient. Therefore, it would be more appropriate to assume a combination of the two models, suggesting that aerosol size distributions and compositions of both conditions may be mixed in the transition from non-Saharan dust to Saharan dust episodes. Even though we chose refractive indices according to the literature, there is still work to be done on aerosol refractive indices. However, a wrong assumption of the refractive index in the models would only yield a systematic increase or decrease in the maximum of the size distribution modes and would not alter the general trend.

\section{Discussion}

Uncertainties in the inversion problem depend also on the shape of the actual aerosol size distributions in the atmosphere. Our concern is that the Saharan dust size distributions have many large particles and the finite size range used in the inversions may lead to retrievals with a large particle tail considerably truncated. To see the influence of this limited size range, we proceed as follows. In Sect. 5.1 we computed the extinction coefficients from known aerosols size distribution. Now, starting from these extinction coefficients we do the inversion but with a size range limited from 20 to $3000 \mathrm{~nm}$ obtaining the corresponding aerosol size distributions and comparing them with the original ones. This "simulation" have been done for desert models of Sect. 5.1 using the refractive index of $1.55-0.005 i$. The results were the following: 1) The retrieved volume size distribution consists of only one mode for the "desert with wind carrying dust" model, masking the multimodal nature, coarse and dust modes, of the size distribution. 2) The retrieved volume size distribution for 
the "desert background" model was accurate up to particles with a radius of $1000 \mathrm{~nm}$. 3) For both size distributions, representing "desert with wind carrying dust" and "desert background", the retrieved volume was truncated for radii larger than $3000-4000 \mathrm{~nm}$ and the accumulation mode never appeared (it was absorbed by the coarse mode).

The uncertainties found in the simulation can be taken as the superior limit for the uncertainties in the retrieved size distribution at Teide Observatory of Fig. 9. Obviously, the dust load considered in the simulation models is much larger than the dust load measured at Teide Observatory. On the other hand, Saharan dust travels a long distance and the largest dust particles settle down before reaching the observatory. Therefore, only a small percentage of the largest dust particles will reach the observatory. For this reason, the retrievals show clearly two modes at Teide Observatory while only one mode appears in the simulation. This means that the coarse mode at Teide Observatory is not large enough to mask the accumulation mode as happened in the simulation. We conclude that the retrieved size distributions at Teide Observatory have smaller uncertainties than was found in the simulation. However, it is possible that the actual size distributions at Teide Observatory have the larger particle mode shifted to larger sizes. Considering the simulation result as an upper limit for the uncertainty, the maximum of the larger particle mode could be higher but not more than $25 \%$, and the mode could be shifted $30 \%$ to larger sizes (maximum limit). The retrieved size distributions presented here can be consider as a model of Saharan and non-Saharan size distributions at Teide Observatory for calculating the extinction coefficients at different wavelengths in the interval from $450 \mathrm{~nm}$ to $870 \mathrm{~nm}$ with an uncertainty that depends on the error of the linear fits in Table 3 . Also, they can be used to calculate aerosol optical properties that have little sensitivity to the uncertainties in the inversion method, for example, the asymmetry factor, which is an aerosol parameter needed in aerosol climate forcing calculations (Gonzalez Jorge \& Ogren 1996).

\section{Conclusions}

The atmospheric extinction over Teide Observatory is very low (between 0.04 and $0.07 \mathrm{mag}$ airmass $^{-1}$ at $680 \mathrm{~nm}$ ) making the observatory a first-class site for astronomical observations. Occasional presence of windborne Saharan dust that may occur during the summer produces a grey effect in the atmospheric extinction in the wavelength range analysed in this work $(450 \mathrm{~nm}$ to $870 \mathrm{~nm}$ ). The extinction then may vary between 0.075 to $0.8 \mathrm{mag}$ airmass $^{-1}$ at $680 \mathrm{~nm}$. The effect of the dust starts to arise when the atmospheric extinction coefficient at $680 \mathrm{~nm}$ is higher than $0.075 \mathrm{mag}$ airmass ${ }^{-1}$. The functions $K_{\lambda_{1}}=f\left(K_{680}\right)$ are straight lines with different behaviour depending on the sky conditions. On clear days (no dust)

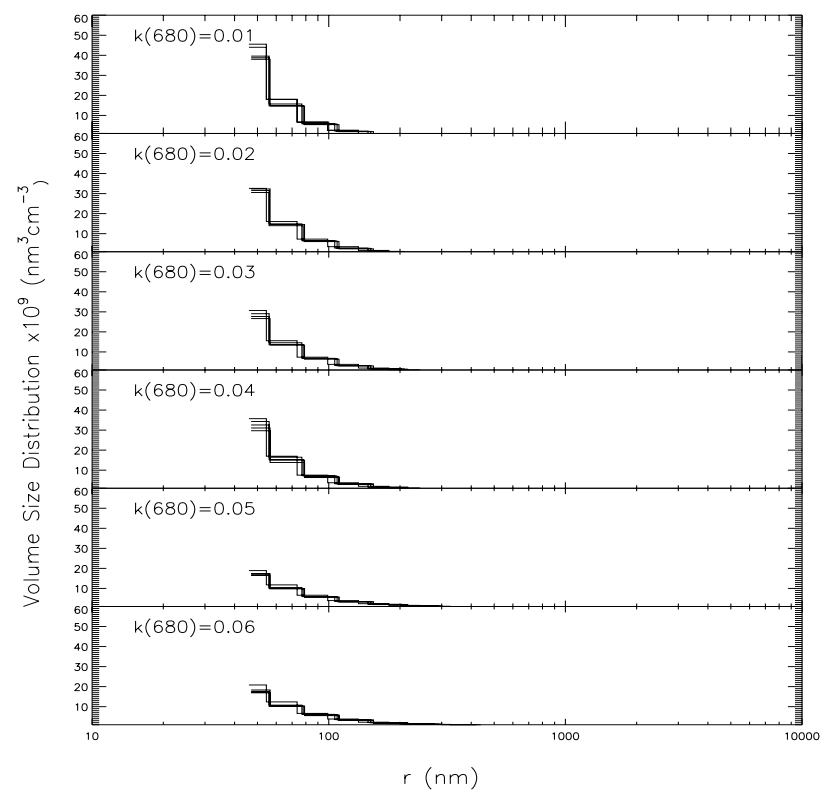

Fig. 8. Aerosol volume size distributions retrieved from the extinction coefficients at Teide Observatory for non-Saharan dust episodes (clear days). The input data were obtained from the linear fits to the real data (Fig. 5a). See text

the slopes of the straight lines are different as expected from pure aerosol atmospheric components following the Ångstrom criterion. For dusty conditions, those slopes are equal to unity (within the errors) indicating that the extinction changes the same quantity in all the wavelengths analysed here (from $450 \mathrm{~nm}$ to $870 \mathrm{~nm}$ ). The retrieved aerosol size distributions at Teide Observatory during nonSaharan dust episodes (the greater part of the year) only show the accumulation mode with a concentration of particles of radius between 40 and $120 \mathrm{~nm}$. These particles are responsible of the wavelength dependence of the atmospheric extinction in the wavelength range of this work. The absence of extinction coefficients at shorter wavelength makes it impossible to compute the aerosol size distribution below $40 \mathrm{~nm}$. When Saharan dust arrives and the atmospheric extinction increases, the retrieved aerosol size distributions show a second mode. The accumulation mode decreases and spreads to higher particle radii indicating that particles of these sizes are being added to the background. The coarse mode maximum appears around 1000 to $2000 \mathrm{~nm}$ and increases as the extinction increases. These particles are responsible of the change in the atmospheric extinction behaviour towards the grey behaviour found in our data. There are two main limitations in the inversion method to retrieve the aerosols size distributions presented in this work. The observed wavelengths from $450 \mathrm{~nm}$ to $870 \mathrm{~nm}$ limit the particle sizes to $20 \mathrm{~nm}$ to 


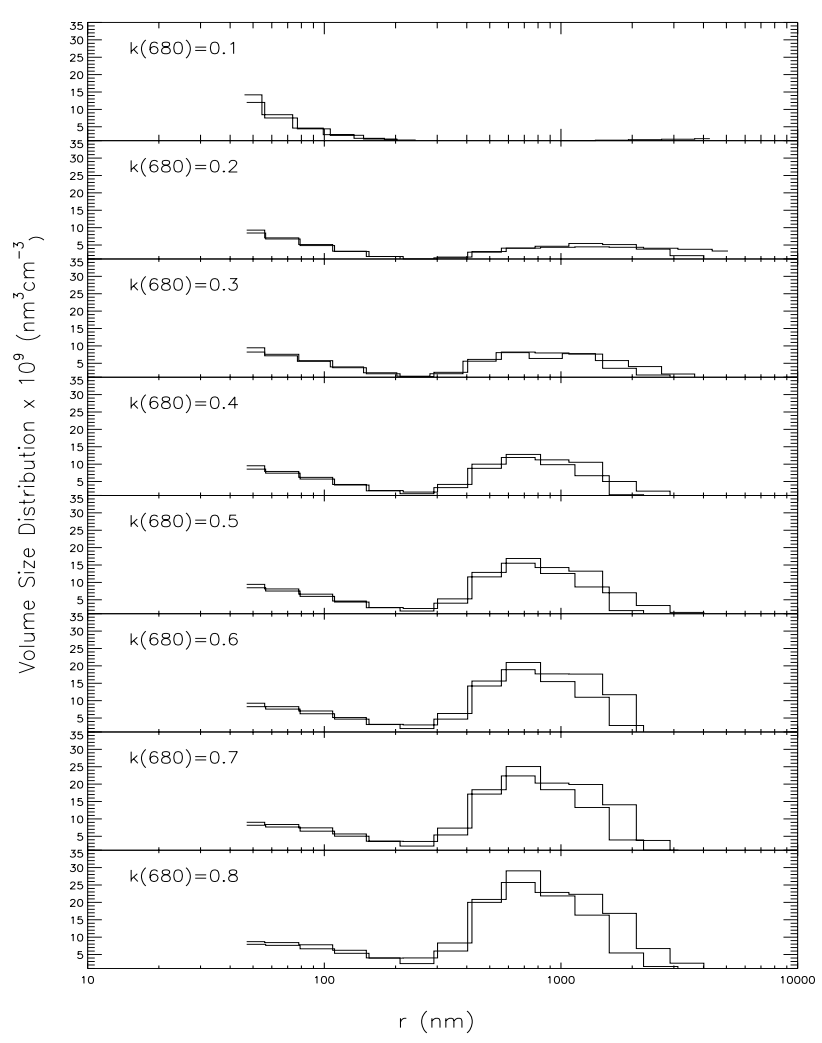

Fig. 9. Aerosol volume size distributions retrieved from the extinction coefficients at Teide Observatory for Saharan dust episodes (dusty days). The input data were obtained from the linear fits to the real data (Fig. 5b). See text

$3000 \mathrm{~nm}$ and the extrapolation of the five wavelengths simultaneously from the straight lines of Fig. 5. For these two reasons, the allocation of the coarse mode maximum during Saharan dust episodes could be shifted to higher radius no more than $30 \%$ as deduced from the simulations. The retrieved size distributions presented here can be considered as a model of Saharan and non-Saharan dust size distributions at Teide Observatory.

For a more complete study a new observing campaign extending the wavelength range would be necessary over a sufficient time span to get a reasonable sample of clear and dusty days (at least two or three years).

Acknowledgements. The help of the maintenance service at the IAC during all the observing periods for this work is gratefully acknowledged. We would also like to express our deep gratitude to all the observers of the helioseismology group at the IAC who patiently took part in the careful observations over these years. We thank the Space Science Department at ESTEC for the development and use of SLOT, especially S. Korzennik and V. Domingo. M.C. Rabello-Soares is also grateful for partial support received from the Brazilian Institution CNPq. Finally, the authors wish to thank the Spanish CAICYT for financial support under grants ESP90-0969 and PB91-0530.

\section{References}

Andersen B.N., Domingo V., Kornennik S., et al., 1988a, in Seismology of the Sun and Sun-Like Stars, Rolfe E.J. (ed.), ESA, Paris Cedex, p. 385

Andersen B.N., Domingo V., Jiménez A., et al., 1988b, SPh 116,391

Brandt P.N., Wohl H., 1982, A\&A 109, 77

Cachorro V.E., Gonzalez M.J., de Frutos A.M., Casanova J.L., 1989, Atmos. Environ. 23, 265

Carlson T.N., Benjamin S.G., 1980, J. Atmos. Sci. 37, 193

Charlson R.J., 1978, Atmos. Environ. 12, 39-53

D'Almeida G., 1987, J. Geophys. Res. 92, D3, 3017-3026

Fouquart Y., Bonnel B., Chaoui Roquai M., Santer R., Cerf A., 1987, J. Climate Appl. Meteor. 26, 28

Golay M., 1974, Introduction to Astronomical Photometry (Astron. Space Sci. Library, Vol. 41). Reidel, Dordrecht

Gonzalez Jorge H., Ogren J.A., 1996, J. Atmos. Sci. 53, 3669

Harvey J.W., 1986, in IAU Sym. 123, Advances in Helio-andAsteroseismology, Christensen-Dalsgaard J. \& Frandsen S. (eds.). Reidel, Dordrecht, p. 497

Heintzenberg J., 1978, Bitr. Phys. Atmos. 51, 91

Heintzenberg J., Muller H., Quenzel H., Thomalla E., 1981, App. Opt. 20, 1308

Hill F., Fischer G., Grier J., et al., 1994, SPh 152, 351

Hill F., et al. (36 authors), 1994, SPh 152, 351

Jiménez A., Pallé P.L., Roca Cortés T., Domingo V., Korzennik S., 1987, A\&A 172, 323

Jiménez A., Pallé P.L., Roca Cortés T., Domingo V., 1988, A\&A 193, 298

Jiménez A., Alvarez M., Andersen B.N., Domingo V., Jones A., Pallé P.L., Roca Cortès T., 1990, SPh 126, 1

King M.D., Byrne D.M., Herman B.M., Reagan J.A., 1978, J. Atmos. Sci. 35, 2153

McInnes B., Walker M.F., 1974, PASP 86, 529

Muñoz-Tuñon C., Vernin J., Varela A.M., 1997, A\&A (in press)

Murdin P., 1985, Vistas Astron. 28, 449

Palmer K.F., Williams D., 1975, Appl. Opt. 14, 208

Patterson E.M., 1977, Appl. Opt. 16, 2414

Sanchez F., 1970, Urania 271, 195

Vernin J., Muñoz-Tuñon C., 1992, A\&A 257, 811

Vernin J., Muñoz-Tuñon C., 1994, A\&A 284, 311

Whitby K.T., 1978, Atmos. Environ. 12, 135

Whittet D.C.B., Bode M.F., Murdin P., 1987, Vistas 30, 135

Wiscombe W.J., 1980, Appl. Opt. 19, 1505 